\title{
2009 Research Day winners
}

A w ide variety of research is conducted by medical students and other undergraduate health science students at Dalhousie. Much of this research is presented each year at Dalhousie's Undergraduate Health Science Student Research Day. The abstracts included here were selected for awards from the more than forty that were presented on February 11, 2009.

Underutilization of implantable cardioverter-defibrillators after coronary artery bypass grafting in patients with impaired left ventricular function

Ryan Kelly, Olivier Heimrath, Karen Buth, Magdy Basta, Jean-Francois Légaré

OBJECTIVE: Implantable cardioverter defibrillator (ICD) therapy is currently utilized to reduce the rate of sudden cardiac death in patients with ischemic coronary artery disease (CAD). The objective of this study was to identify and examine pre-operative predictors of ICD implantation in a large group of eligible coronary artery bypass grafting (CABG) patients with impaired left ventricular function. METHODS: We conducted a retrospective cohort study of ICD eligible patients who underwent any CABG between March 1, 1995 and June 30, 2008 at the Queen Elizabeth II Health Sciences Center in Halifax Nova Scotia. Patients who had undergone CABG surgery with pre-operative left ventricular ejection fraction (LVEF) of $\leq 35 \%$ were included. Patients with an existing ICD were excluded. The event of interest was ICD implantation. RESULTS: A total of 1,169 out of 11,931 CABG patients had LVEF $\leq 35 \%$ and were defined as ICD eligible (mean $\mathrm{EF}=27.3 \%+/-6.4 \%$ ). Of these eligible patients, only $101(8.6 \%)$ received an ICD after CABG. The median time to implant was 255 days (IQR $=14$ - 1078 days). The single variable that independently predicted eventual ICD implantation was a history of pre-operative arrhythmia $(\mathrm{OR}=7.4 ; \mathrm{Cl}, 4.4-12.2)$. The variables that predicted not having an ICD implanted after $\mathrm{CABG}$ included requiring an urgent or emergent surgery $(\mathrm{OR}=$ $0.5 ; \mathrm{Cl}, 0.2-0.9)$, age $>70$ years $(\mathrm{OR}=0.5 ; \mathrm{Cl}, 0.3-0.8)$, female gender (OR $=0.2 ; \mathrm{Cl}, 0.1-0.6)$, or having COPD (OR $=0.5$; $\mathrm{Cl}, 0.3$ - 0.8). CONCLUSION: Despite the fact that implantation rates are on the rise, ICD therapy appears underutilized among CABG patients with impaired left ventricular function. This fact is particularly evident among females and elderly patients.

Immunohistochemical investigation of intraspinal neurons expressing the serotonergic transporter using transgenic mice

Natalie Parks, Robert Brownstone

BACKGROUND: In mammals serotonergic neurons are concentrated in the brainstem raphe nuclei, extending axonal projections into the spinal cord. An extraordinarily small number of intraspinal serotonergic neurons have been observed in the adult rat (5-8) - failing to account for levels of serotonin observed following spinal cord transection. Abundant $\mathrm{GFP}^{+} / 5-\mathrm{HT}^{-}$cells in the spinal cord of adult SERT ${ }^{\text {cre/+x} T h y 1-f s-Y F P(l i n e 15) ~ m i c e, ~}$ expressing cre recombinase promoted by the serotonin transporter (SERT), led to the hypothesis that $\mathrm{GFP}^{+}$intraspinal cells synthesize serotonin early in development. METHODS: Spinal cords from E14 and E16 SERT ${ }^{\text {cre/+x} T h y 1(l i n e 15) ~ m i c e ~ w e r e ~ t r e a t-~}$ ed immunohistochemically and examined using a confocal laser scanning microscope. RESULTS: $\mathrm{GFP}^{+}$cells were present at all levels of the E14 and E16 spinal cord with a higher density of $\mathrm{GFP}^{+}$cells at E14. All $\mathrm{GFP}^{+}$cells were neurons, however, none were serotonergic during the embryonic stages examined. Neurons were of varied morphology including cells in the ventral horn motor column of similar morphology as $\alpha$-motoneurons. CONCLUSIONS: Despite claims that the SERT ${ }^{\text {cre/t+ }}$ strain exhibits specificity based on neurotransmitter status, GFP $/ 5-\mathrm{HT}^{-}$cells have now been demonstrated in the adult thalamus, cingulate cortex, hippocampus, and lumbar spinal cord. Therefore, YFP (and hence SERT) expression is not a marker exclusively for serotonergic neurons. Decreasing density of $\mathrm{GFP}^{+}$cells with development suggests that SERT expression in non-serotonergic neurons may be important for normal spinal cord development. Future studies will include in situ hybridization for SERT to confirm that YFP expression is promoted by SERT in $\mathrm{GFP}^{+}$neurons that never express serotonin.

The role of endothelin-1 in rat optic nerve head astrocyte proliferation: Implications for glaucoma

Jeremy Murphy, Michele Archibald, William Baldridge, Balwantray Chauhan

BACKGROUND: Glaucoma, the second leading cause of blindness, is characterized by remodeling of the optic nerve head which leads to astrocyte migration and proliferation, and subsequently results in a progressive loss of retinal ganglion cells. The goal of this research is to characterize endothelin-1's (ET-1) influence on rat optic nerve head astrocyte $(\mathrm{ONHA})$ proliferation. METHODS \& RESULTS: ONHAs were isolated from adult rats and characterized using immunohistochemistry. These cells all express GFAP (mature astrocyte marker), and both ET-1 receptors (ETB and ETA), but do not label for MBP (mature oligodendrocyte marker) or for A2B5 (astrocytes outside the ONH). To determine if ET-1 influenced ONHA proliferation, cells were either untreated, treated with vehicle, or with ET-1 (at 10-6, 107, $10-9$ or $10-11 \mathrm{M})$. Cell numbers did not change 1 day after treatments; however, a significant increase occurred at 2 days following 10-6, 10-7, 10-9 M ET-1 doses (by 18\%, 33\%, and $24 \%$ respectively) but not $10-11 \mathrm{M}$ ( $\mathrm{n}=4$ /group). Three days after treatment, cell numbers increased significantly at all doses $(88 \%$, $77 \%, 99 \%, 97 \%$ for 10-6, 10-7, 10-9 and 10-11 M respectively). To determine whether Et-1 induced proliferation through ETB or ETA we investigated ONHA proliferation 2 days following ET-1 (at 10-7 or 10-9 M) exposure with BQ-788 (ETB antagonist) or BQ-610 (ETA antagonist). No proliferation occurred in the presence of either antagonist. CONCLUSIONS: These findings indicate that (1) purified ONHAs may be isolated from rodents, (2) these cells proliferate with ET-1 exposure, and (3) this proliferation requires both ETA and ETB. (Supported by Dalhousie University Medical Student Research Fellowship (JM) and Canadian Institutes of Health Research) 
Rapid influenza virus detection for laboratories in remote areas

Stephen Smith, Todd Hatchette, Janet Mills, Jason Leblanc

BACKGROUND: Antiviral drugs active against influenza can reduce morbidity and mortality if initiated within 48 hours of onset of clinical illness. We evaluated a commercially available realtime reverse transcriptase-PCR (RT-PCR) assay for influenza diagnosis that provides results within three hours. The assay is low throughput and kit-based, making it appealing for laboratories in remote areas. METHODS: Ninety-seven consecutive nasopharyngeal specimens submitted for testing during the 2007 season were tested for influenza virus by three methods: 1) a real-time RT-PCR using the Flu A/B r-gene Primer/Probe mix (Argene, Varilhes, France) performed on the Spartan DX Realtime DNA Analyzer (Spartan Bioscience, Ottawa, Ontario); 2) the Influenza A\&B Respi-Strip (Coris BioConcept, Belgium) rapid membrane antigen capture test; and 3) a previously validated conventional RT-PCR assay. Analytical sensitivity of the Spartan $D X /$ Argene assay was assessed using both extracted influenza A virus RNA and a DNA construct of the target sequence of the assay. Analytical specificity of the Spartan DX/Argene system was determined using nucleic acid extracts of various other respiratory pathogens and avian influenza viruses. RESULTS: When compared to conventional RT-PCR, the clinical sensitivity of the Spartan DX/Argene real-time RT-PCR assay (84.4\%) outperformed the Respi-Strip rapid antigen tests (24.4\%). A total of 31 of $35(88.6 \%)$ specimens identified as influenza A-positive and 7 of $10(70.0 \%)$ specimens identified as influenza B-positive by the conventional RT-PCR protocol also tested positive using the Spartan DX/Argene system. Only $5(14.3 \%)$ of the influenza A-positive specimens and $6(60 \%)$ of the influenza B-positive specimens tested positive using Respi-Strip. Clinical specificity of both techniques was $100 \%$ relative to conventional RT-PCR. The Spartan DX/Argene real-time assay was able to detect avian influenza virus subtypes $\mathrm{H} 1$ to $\mathrm{H} 13$ and produced negative results upon testing of nucleic extracts of a variety of non-influenza respiratory pathogens. However, the analytical sensitivity of the real-time RT-PCR assay was shown to be approximately 100fold less sensitive than conventional RT-PCR. CONCLUSIONS: Direct antigen testing of influenza virus using assays such as Respi-Strip is rapid, yet poor sensitivity precludes their routine clinical use. The combined use of the Spartan DX Real-time DNA Analyzer and the Argene Flu A/B r-gene Primer/Probe mix generates results in less than three hours and eliminates many of the barriers that preclude introduction of real-time PCR methods in small laboratories. However, further optimization of the assay is necessary to enhance sensitivity.

Reliability and validity of a functional magnetic resonance imaging (fMRI) task paradigm for the prediction of languageassociated regions of the frontal lobe

Sarah Ironside, David Clarke, Gail Eskes

PURPOSE: To measure the reliability and validity of an fMRI language task to be used for presurgical brain mapping in patients with brain tumors. As a first step, this study aimed to determine the degree of consistent fMRI activation in the left inferior frontal gyrus (Broca's area) during a verbal fluency task in ten healthy controls, followed by application of the language task paradigm to the pre-operative assessment of neurosurgical patients. METHODS: Ten control participants (mean age 25.6), were scanned at two time points one week apart in a 1.5T MRI scanner during a verbal fluency task. Patients involved in the study received one pre-operative scan followed by awake surgery with electro-cortical stimulation mapping to confirm the location of language cortex in the frontal lobe. RESULTS: Reliable fMRI activation in Broca's area was observed for each of the ten control participants across two scanning sessions. Participants generated significantly more words and had shorter, faster reaction times to the first word generated per task block during the second scanning session. The issues in comparison of the localization results from this task paradigm with intraoperative language mapping in neurosurgical patients will be discussed. CONCLUSIONS: Results indicate that verbal fluency is a reliable task for localizing expressive language regions with fMRI. Furthermore, the task shows promise as a tool for presurgical assessment of language function in neurosurgical patients. (Funding provided by Music-In-Medicine Summer Studentship over the period June - August 2008)

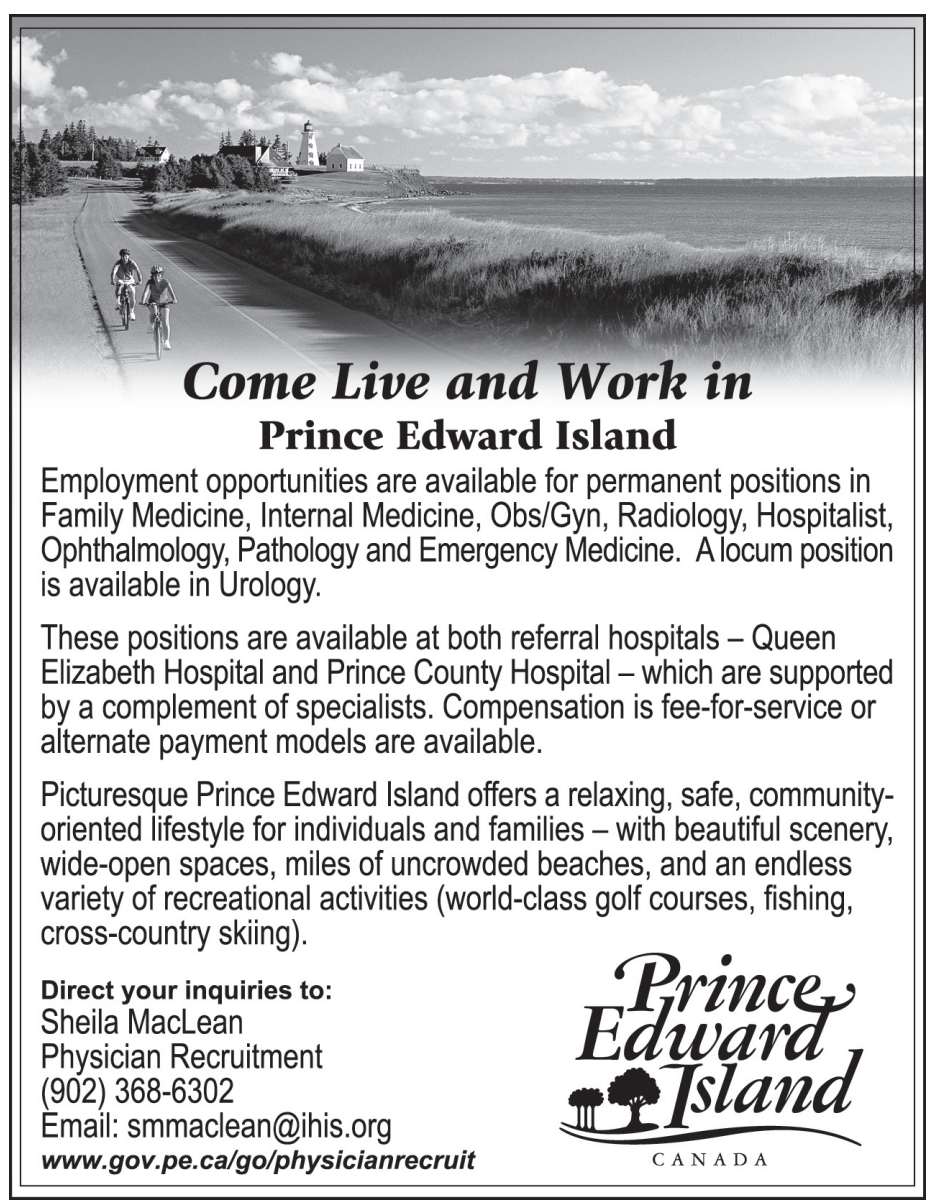




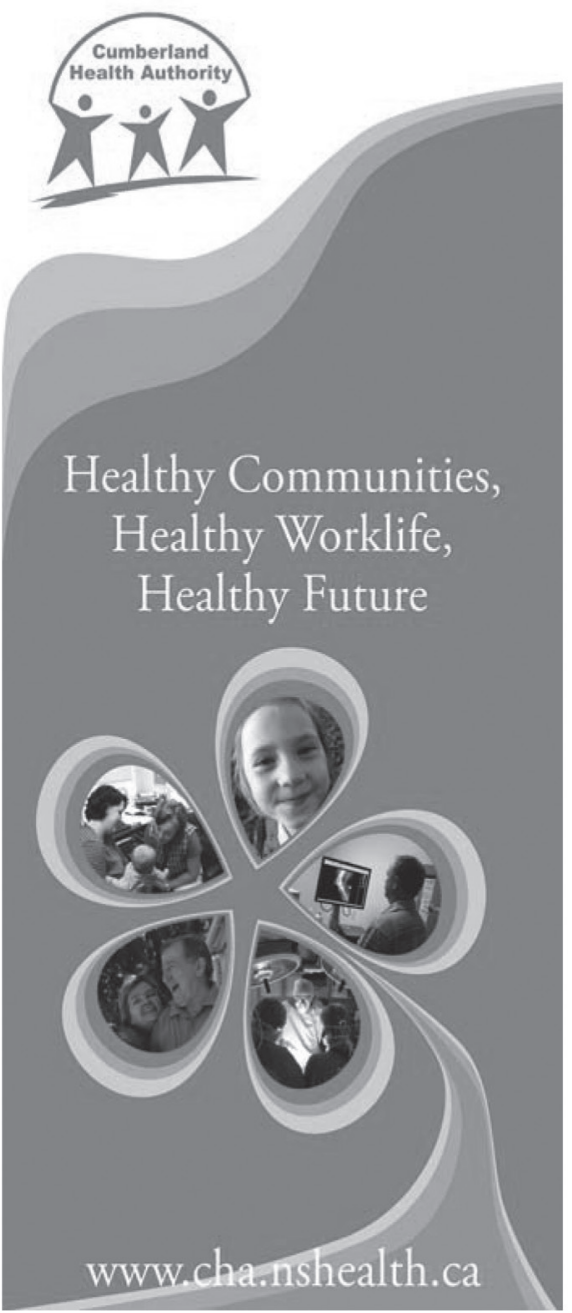

Cumberland County

Nova Scotia ... A Great Place To Live \& Work

Located in the centre of the Maritimes, we offer a variety of specialist opportunities in a state-of-the art acute care setting or you can become part of innovative primary care practices.

For more information, please contact cha_humanresources@ cha.nshealth.ca or call our offices at 902-661-1090

Find us on-line at www.cha.nshealth.ca

\section{"MCI takes care of everything} without telling me how to run my practice."

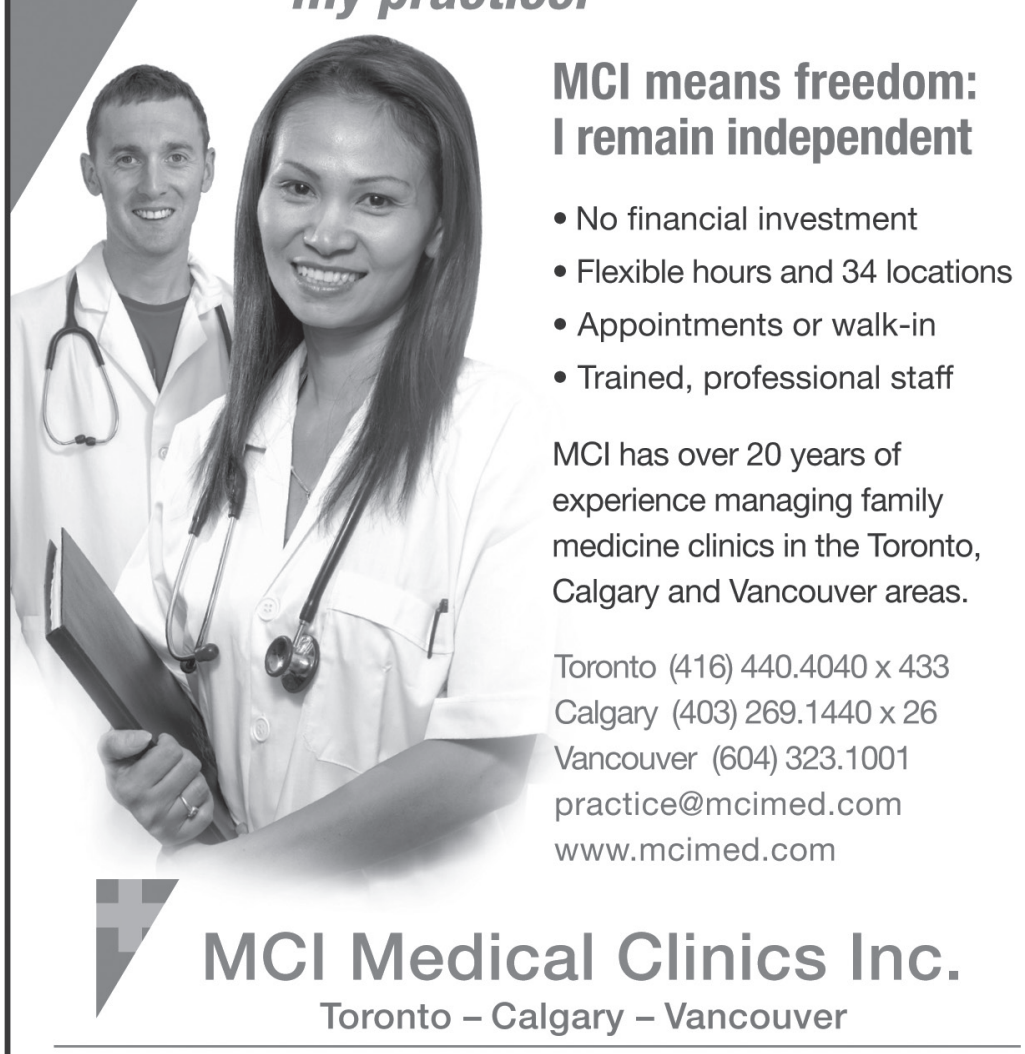

Works for Doctors - Cares for Patients - Empowers Employees

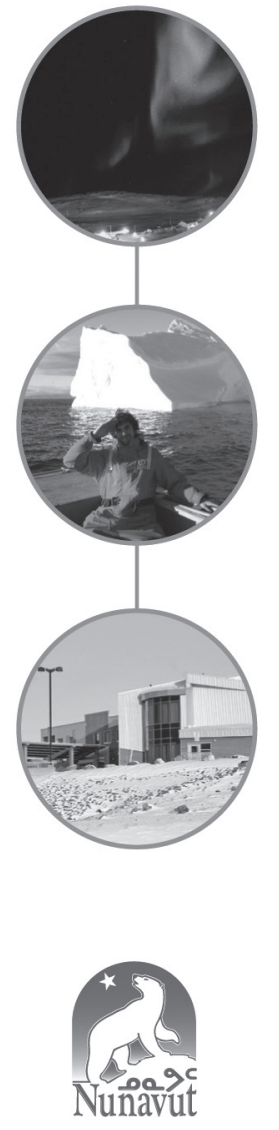

family

practitioners opportunities in Nunavut

This is your unique opportunity

to experience rich Inuit culture,

breathtaking scenery, and

a change of pace.

As a family practitioner in Nunavut, you will have a unique opportunity to shape the development of healthcare in Nunavut. You will combine clinical practice with community visits, working in a collaborative environment with other front-line healthcare providers and liaising with support services in Iqaluit, Winnipeg. Yellowknife and Ottawa.

If you are a dynamic family physician with a sense of adventure, strong leadership skills, and a desire to make a difference to healthcare in the north, you will thrive in this exciting environment.

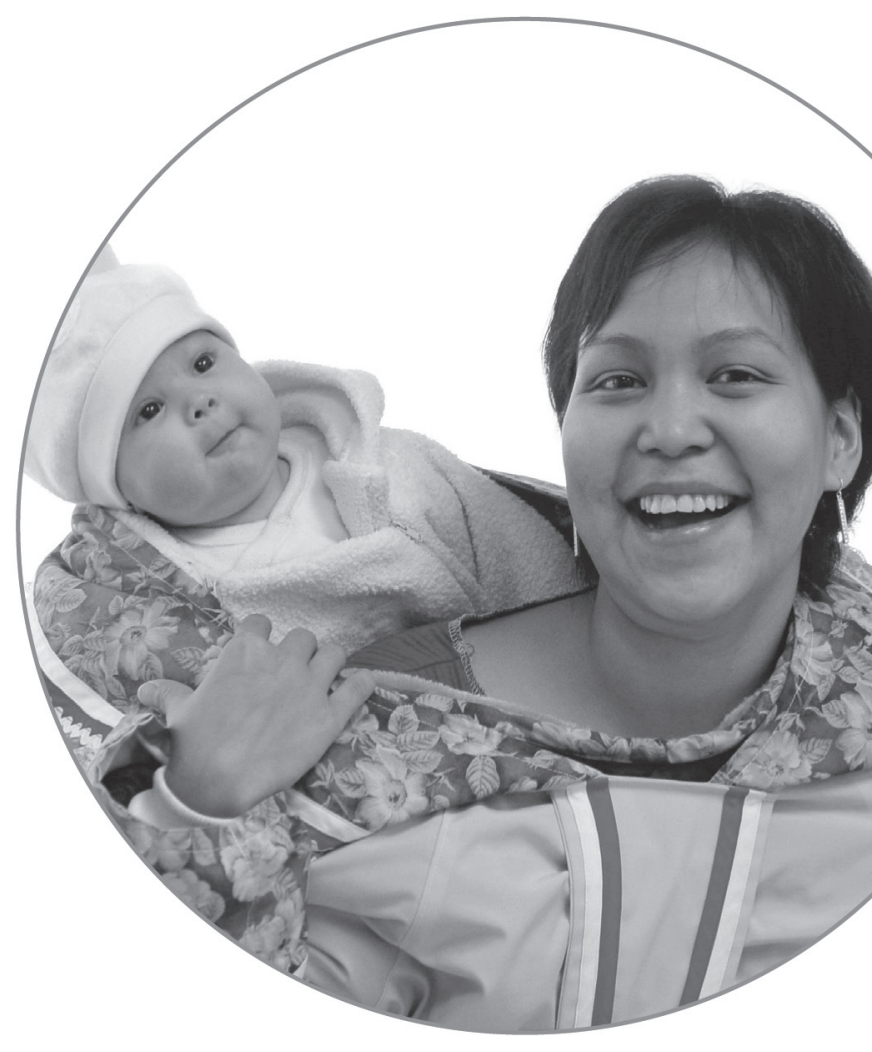

For more information, please contact Physician Services: medicine@gov.nu.ca • 877.979.7343 